\title{
Primary Pleural Synovial Sarcoma with Metastatic Cardiac Involvement: A Case Report
}

\author{
Ji-Hye Won, ${ }^{1}$ Susie Chin, ${ }^{2}$ Jai Soung Park, ${ }^{1,}$ Sang Hyun Paik, ${ }^{1}$ Heon Lee, ${ }^{1}$ Jang Gyu Cha, ${ }^{1}$ Hwa Kyoon \\ Shin, ${ }^{3}$ and Eun Suk Koh ${ }^{2}$ \\ ${ }^{1}$ Department of Radiology, Bucheon Hospital, Soonchunhyang University College of Medicine, Bucheon, South Korea \\ ${ }^{2}$ Department of Pathology, Bucheon Hospital, Soonchunhyang University College of Medicine, Bucheon, South Korea \\ ${ }^{3}$ Department of Thoracic Surgery, Bucheon Hospital, Soonchunhyang University College of Medicine, Bucheon, South Korea \\ "Corresponding author: Jai Soung Park, Department of Radiology, Bucheon Hospital, Soonchunhyang University College of Medicine, Bucheon, South Korea, E-mail: \\ jspark@schmc.ac.kr
}

Received 2016 July 25; Revised 2016 August 19; Accepted 2016 August 24.

\begin{abstract}
Primary pleuropulmonary synovial sarcomas are rare soft tissue malignancies; combined metastatic involvement of the heart is extremely rare. In this case report, a 17-year-old female presented with a history of chest pain. Chest radiographs revealed a round mass in the left upper hemithorax, and computed tomography (CT) showed a well-defined heterogeneous enhancing mass abutting the pleura. A core needle biopsy revealed malignant spindle cells. Surgical resection was performed, and a final diagnosis of primary pleural synovial sarcoma, monophasic fibrous type, was made. The patient underwent radical irradiation and chemotherapy and remained stable for 28 months until a follow-up chest CT showed a poorly enhancing nodule in the left pericardial region that enlarged after 5 months. Surgical resection was performed. Histological examination confirmed metastatic cardiac involvement from a primary pleural synovial sarcoma. We report this unusual case of a primary pleural synovial sarcoma metastasis to the heart.
\end{abstract}

Keywords: Sarcoma, Metastasis, Heart, Computed Tomography (CT)

\section{Introduction}

Primary pleuropulmonary synovial sarcoma (PPSS) is a rare soft tissue malignancy representing only $0.1 \%-0.5 \%$ of all primary lung malignancies. It commonly arises from the chest wall, lung parenchyma, pleura or mediastinum. It is usually diagnosed during the fifth decade of life (1). The frequency of cardiac metastasis from soft tissue sarcomas ranges from $3.5 \%$ to $35 \%$ with only two cases of metastatic synovial sarcoma of the heart reported: one involving the right ventricle and the other the left ventricle. Both cases had a primary tumor in the extremities rather than in the lungs without focusing on the imaging findings of these rare lesions (2-4). We report a case of PPSS that eventually metastasized to the heart in a 17-year-old female.

\section{Case Presentation}

A17-year-old female presented with dull pain in the left upper chest alternating with brief periods of chest discomfort lasting a few months. She and her parents were nonsmokers with no history of radiation or chemical exposure. She had no serious medical or surgical history and grew up in normal residential and social environments. She had no shortness of breath or cough. There was no history of weight loss, fever, or night sweats. Her vital signs and physical examination were unremarkable. All laboratory data including blood tests, standard biochemical tests, and urinalysis were within normal limits.

Chest radiographs (Figure $1 \mathrm{~A}$ and $\mathrm{B}$ ) revealed a welldefined round mass in the left upper hemithorax. The mass was at an obtuse angle to the chest wall and was approximately $8 \mathrm{~cm}$ long. There was no other abnormality in the lung parenchyma or bony thorax.

Chest CT (Figure 1C - E) confirmed a well-defined ovalshaped mass with heterogeneous enhancement in the left upper hemithorax abutting the pleura. In the pre-contrast image, the lesion showed homogeneous soft tissue attenuation and a lack of calcification, but a small amount of high-attenuation fluid was present in the left pleural space, suggestive of hemothorax. After contrast enhancement, the mass showed heterogeneous enhancement, with attenuation similar to that of the back muscles, and contained subtle low densities suggestive of necrosis or hemorrhage. There was no evidence of adjacent bone destruction or chest wall invasion. The mediastinal and hilar 

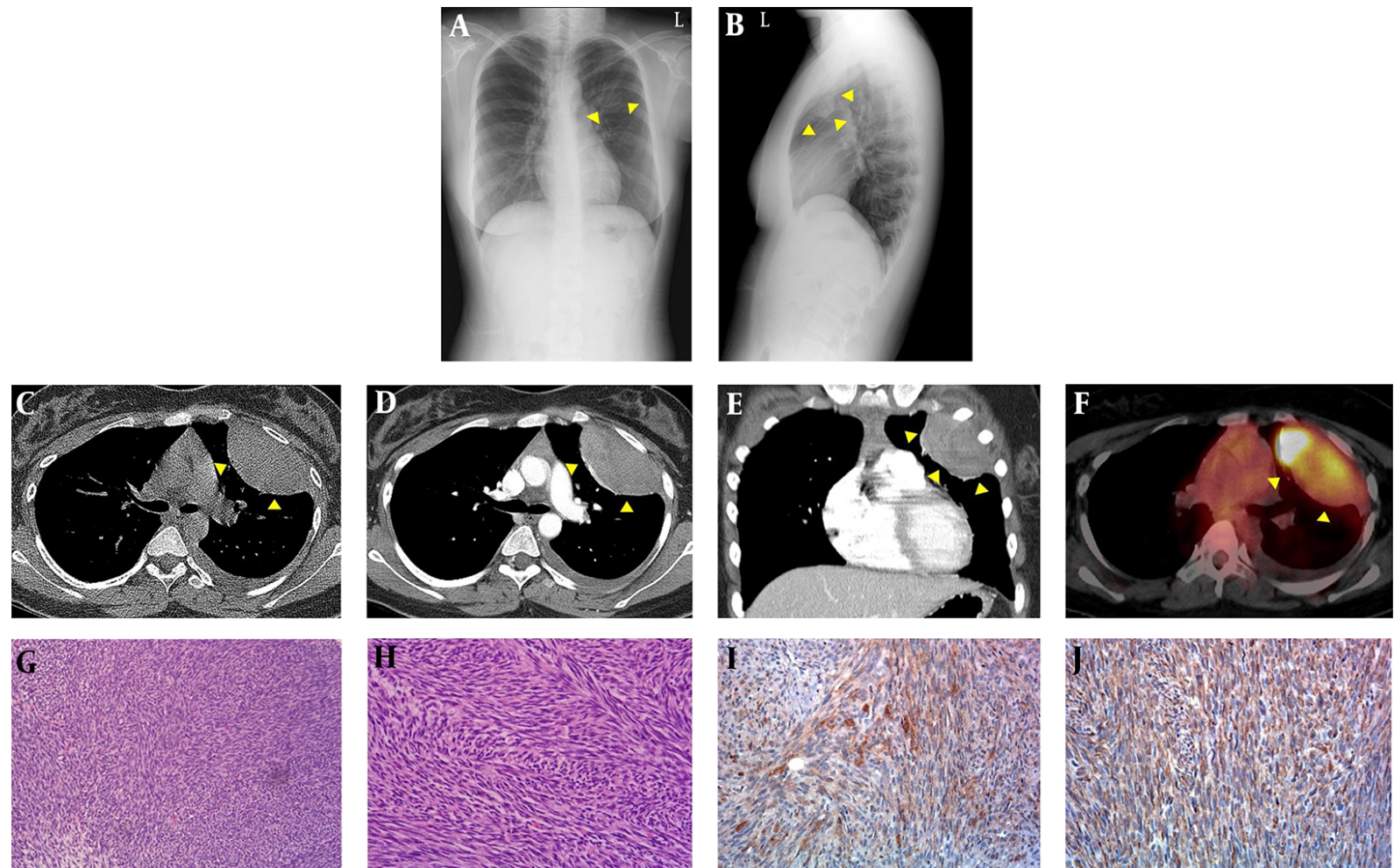

Figure 1. A, B, Chest radiographs; C, chest CT; D, positron emission tomography (PET)-CT; E - G, microscopic features of primary pleuropulmonary synovial sarcoma (PPSS) in a 17-year-old female. A posteroanterior; B, left lateral chest radiographs show a well-circumscribed round mass (arrowheads in A and B) in the left upper hemithorax; C, precontrast; D, contrast-enhanced CT axial; E coronal images of the chest show a well-circumscribed, oval-shaped mass (arrowheads in C, D and E) with heterogeneous enhancement abutting the pleura in the left upper hemithorax and a small amount of high-density fluid in the precontrast image; F, axial F-18 fluorodeoxyglucose positron emission tomography-CT (18F FDG PET/CT) shows increased FDG uptake in the mass (arrowheads in F), with a maximum standardized uptake value of 31.7; G, the tumor is composed of tightly packed fascicles of spindle cells (hematoxylin and eosin $(\mathrm{H}-\mathrm{E})$ staining, $\times 100)$; $\mathrm{H}$, a fibrosarcoma-like area showing distinct intersecting fascicles (a herringbone pattern) is also noted (H - E staining, $\times 200$ ); I, patchy expression of epithelial membrane antigen (EMA) is evident (EMA staining, $\times 200$ ); J, the tumor expressed CD99 in a diffuse cytoplasmic staining pattern (CD99 staining, $\times 200)$.

lymph nodes were not enlarged. In the scanned portion of the abdomen, there were no masses, lymphadenopathy, or ascites.

F-18 fluorodeoxyglucose positron emission tomography-CT (18F FDG $\mathrm{PET} / \mathrm{CT})$ revealed increased FDG uptake in the mass, with a maximum standardized uptake value of 31.7 (Figure $1 \mathrm{~F}$ ).

From these imaging findings, the diagnoses of a localized fibrous tumor of the pleura, malignant mesothelioma, metastatic pleural malignancy, and rare primary pulmonary sarcoma (e.g., pleuropulmonary synovial sarcoma, fibrosarcoma, leimyosarcoma, sarcomatoid carcinoma, malignant nerve sheath tumor, hemangiopericytoma, and malignant fibrous histiocytoma) were considered. These diagnoses were based on the tumor's appearance as a sharply marginated, heterogeneously enhanced mass with low attenuation foci and no involvement of the bone or calcifications. CT-guided core needle biopsy of the mass yielded two samples of yellowish gelatinous mate- rial. Microscopic examination showed relatively uniform spindle-shaped cells with occasional mitoses arranged in tight fascicles. The tumor cells were immunoreactive to vimentin and CD99 and were focally positive for epithelial membrane antigen (EMA), suggesting a monophasic synovial sarcoma. Medical and surgical oncologists agreed to treat the tumor with surgical resection and to initiate a combination chemotherapy regimen consisting of five cycles of vincristine, doxorubicin, and cyclophosphamide, alternating with four cycles of ifosfamide and etoposide. A thoracotomy was performed, and the mass was found to arise from the visceral pleura and to adhere to the left first to third intercostal spaces, with invasion into the left first intercostal muscle with adjacent hemothorax. The surgical specimen consisted of an $8.0 \times 6.5 \times 5.5 \mathrm{~cm}$ wellcircumscribed but unencapsulated tumor. The tumor was whitish-yellow, soft, and fleshy with cystic degenerative changes and hemorrhage. There was no calcification in the tumor. Histologically, the tumor was composed of densely 
packed, cellular sheets of spindle cells, and some of the tumor cells were arranged in intersecting fascicles in a herringbone pattern, suggesting fibrosarcoma-like changes (Figure 1G-H). Loose and hypocellular focal areas were found at the periphery of the tumor. The tumor cells were relatively uniform with ovoid nuclei, scant cytoplasm, and occasional mitotic figures. Immunohistochemical staining revealed immunoreactivity of the tumor cells to cytokeratin, EMA, and CD99 but not to CD34 or desmin (Figure1IJ). A final diagnosis of a monophasic synovial sarcoma from the visceral pleura was made based on the histological and immunohistochemical findings. She was discharged after surgical resection without complications, after which she underwent adjuvant chemotherapy and radiotherapy.

The patient remained stable for 28 months until followup contrast-enhanced chest $\mathrm{CT}$ revealed a $1.6 \mathrm{~cm}$ low attenuation nodule with poor enhancement in the left pericardial area and a small amount of left pericardial and pleural fluid (Figure 2A). Subsequent 18F FDG PET/CT showed slightly increased FDG uptake in the developing nodule with suspected metastasis. Five months after discovery, follow up contrast-enhanced chest CT demonstrated a wellcircumscribed polycyclic marginated mass with heterogeneous enhancement in the left pericardial region that had enlarged from $1.6 \mathrm{~cm}$ to $5.1 \mathrm{~cm}$ (Figure 2B). Although MRI is the most accurate technique for demonstrating cardiac mass, oncologists found the following MRI might be ineffective for choosing further treatment for the pericardial mass due to high cost. They had no choice but to do surgery for the mass whether to perform MRI or not. Additionally, CT also provided proper information for ongoing surgery. Therefore, they agreed to operate surgical resection to treat pericardial mass without obtaining cardiac MRI.

A second mass resection was performed. The tumor specimen was a $6.0 \times 5.0 \times 2.0 \mathrm{~cm}$ well-circumscribed, whitish-yellowish, fleshy to rubbery mass with zones of necrosis and hemorrhage. The tumor was lateral to the left ventricular wall with invasion into the pericardium, myoendocardium, and endocardium of the left ventricle. Microscopically, the tumor contained tight clusters and fascicles of spindle cells with scant cytoplasm, identical to the PPSS, confirming a metastatic synovial sarcoma with left pericardial, myoendocardial, and endocardial involvement.

After metastatectomy, the patient received adjuvant chemotherapy and radiotherapy. The patient's postoperative period was complicated by recurrent pneumonia. Next, hematogenous metastases were detected in the liver, breast, and pleura. The patient died from sepsis 37 months after the initial diagnosis.

\section{Discussion}

PPSS is a rare mesenchymal spindle cell malignancy representing a type of pulmonary sarcoma that comprises $0.1 \%-0.5 \%$ of all primary lung malignancies. Typically, it arises from the chest wall, lung parenchyma, pleura, or mediastinum, but rarely at the level of the bronchial tree, and during the fifth decade of the patient's life (1). There is no significant gender difference in incidence (5). The prognosis for patients with PPSS remains uncertain but is usually poor. The 5-year survival rate varies from $36 \%$ to $76 \%$ (6).

A small but increasing number of cases of PPSS have been reported over the past decades (5), but very few of these cases have involved cardiac metastasis. Cardiac metastasis from soft tissue sarcomas varies from 3.5\% to $35 \%$, with only two cases of metastatic synovial sarcoma of the heart reported: one involving the right ventricle and the other the left ventricle. Both of those cases had a primary tumor in the extremities but not in the lung without focusing on the imaging findings of these rare lesions (2-4). Additionally, a literature review of soft tissue sarcomas included only 105 cases of cardiac metastasis. In that review, the predominant histological type was leiomyosarcoma (22\%) followed by rhabdomyosarcoma (19\%), and liposarcoma (9\%); $27 \%$ of the cases were unspecified. That report suggested that the clinical manifestation of cardiac metastasis can be subtle, as one-third presented with congestive cardiac failure, which led to other complications such as embolism, tumor thrombus, cardiac tamponade, and arrhythmia (2). In our case, the patient was initially asymptomatic except for dull chest pains with only pericardial effusion on follow-up chest CT. Symptoms frequently associated with these tumors are chest pain, dyspnea, and cough; up to $40 \%$ of patients are asymptomatic (7).

The radiological features of PPSS are summarized as follows: chest radiographs typically demonstrate a welldefined pleural-based or parenchymal mass-like lesion occasionally associated with ipsilateral pleural effusion. Generally, the contralateral lung is not involved. CT and magnetic resonance imaging (MRI) are relatively useful tools for evaluating PPSS, although the findings are nonspecific. The most common CT finding of PPSS is a well-defined, heterogeneously enhancing mass often with combined ipsilateral pleural effusion. The inner low-density area often contains necrosis, hemorrhage, or fibrous components. Associated lymphadenopathy as well as calcifications, cortical bone destruction, or tumor infiltration of the chest wall and musculature are infrequent. MRI is useful for assessing the extent of the tumor. On MRI, PPSS often exhibits heterogeneous high signal intensity on T2-weighted images and iso- or low signal intensity on T1-weighted images. After administering gadolinium, PPSS presents as a 

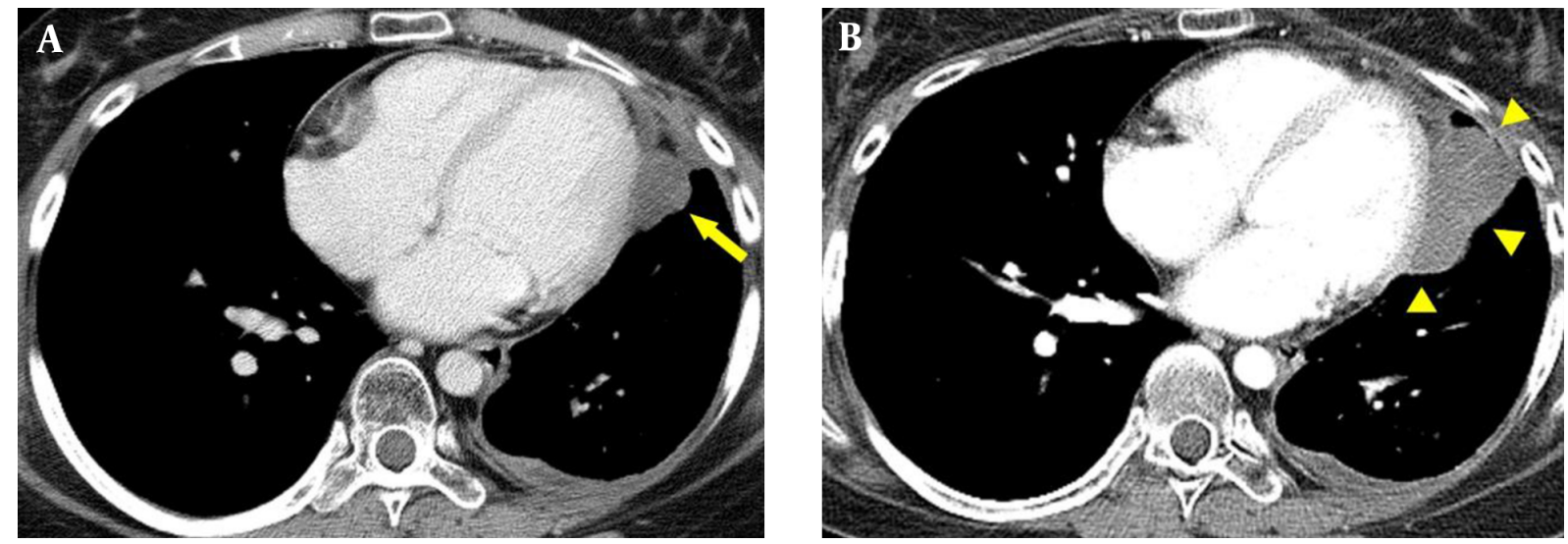

Figure 2. Follow up chest CT (A, B) of the primary pleuropulmonary synovial sarcoma (PPSS) with cardiac metastasis. A, contrast-enhanced axial CT image of the chest shows a poorly enhanced nodule (arrow in A) in the left pericardial region with a small amount of left pericardial and pleural effusion; B, follow up axial contrast-enhanced CT after 5 months shows a well-circumscribed, polycyclic, marginated mass (arrowheads in B) that had increased in size, with heterogeneous enhancement in the left pericardial region.

heterogeneously enhancing mass (5). On PET, PPSS exhibits increased FDG uptake.

Differential diagnoses for PPSS include metastasis from the extrapulmonary synovial sarcoma, a primary tumor such as a pleural fibroma or mesothelioma, or other primary sarcomatous or sarcomatoid neoplasms. The typical clinical and radiologic features can help narrow down the differential diagnosis of PPSS. The presence of hypoglycemia or hypertrophic osteoarthropathy supports a localized fibrous tumor. A history of asbestos exposure and the presence of pleural plaques are indicative of malignant mesothelioma. Mediastinal lymphadenopathy suggests bronchogenic carcinoma rather than PPSS. Metastatic disease rarely manifests as a large solitary pulmonary lesion, although many other rare primary sarcomatous or sarcomatoid neoplasms cannot be distinguished reliably from PPSS based on clinical and radiologic features alone (5).

Histologically, synovial sarcomas are classified as biphasic or monophasic. Monophasic synovial sarcomas are much more common, representing 94\% of PPSS, and are composed of uniform spindle cells. Biphasic synovial sarcomas contain both epithelial and spindle cell components. The surrounding plump spindle cells have hyperchromatic nuclei with scant cytoplasm resembling the tumor cells of monophasic synovial sarcoma. Immunohistochemically, most synovial sarcomas show focal expression of cytokeratin and EMA. Furthermore, 30\% are protein S-100 positive, 60\% - 70\% CD99 positive, and 75\% $100 \%$ Bcl-2 positive (8)

The prognosis of PPSS remains uncertain but is usually poor. The 5-year survival rate varies from 36\% to $76 \%$ depending on the patient's age, tumor size, and tumor resectability (6). There is no recommended treatment for
PPSS, but the mainstay of treatment for all soft tissue sarcomas is surgical resection. Aggressive surgery can reduce the tumor size before or after chemotherapy, and complete surgical excision seems to improve the survival of patients with PPSS. Radiation therapy can also be used to treat PPSS (1). Combination chemotherapy consisting of doxorubicin and ifosfamide has been associated with higher rates of survival in patients with synovial sarcoma (6).

In summary, PPSS is a very rare, aggressive malignancy that must be considered in the differential diagnosis of lung and pleural malignancies. The imaging findings of PPSS are nonspecific, and PPSS is difficult to confirm based on imaging; detailed immunohistochemical staining is required for definitive diagnosis. PPSS with cardiac metastasis is also extremely rare, and only two such cases have been reported. However, cardiac metastasis of PPSS can lead to immediate or delayed death; therefore, early detection and appropriate management are very important to extend survival.

\section{Footnotes}

Authors' Contributions: Study concept and design: JiHye Won, Jai Soung Park and Susie Chin; acquisition of data: Jai Soung Park and Ji-Hye Won; analysis and interpretation of data: Sang Hyun Paik, Susie Chin and Ji-Hye Won; drafting of the manuscript: Heon Lee and Ji-Hye Won; critical revision of the manuscript for important intellectual content: Jai Soung Park, Jang Gyu Cha and Sang Hyun Paik; administrative, technical and material support: Eun Suk Koh, Shin and Susie Chin; study supervision: Jai Soung Park, Sang Hyun Paik and Heon Lee

Financial Disclosure: There is nothing to disclose. 


\section{References}

1. Keel SB, Bacha E, Mark EJ, Nielsen GP, Rosenberg AE. Primary pulmonary sarcoma: a clinicopathologic study of 26 cases. Mod Pathol. 1999;12(12):1124-31. [PubMed: 10619264].

2. Tak T, Goel S, Chandrasoma P, Colletti P, Rahimtoola SH. Synovial sarcoma of the right ventricle. Am Heart J. 1991;121(3 Pt 1):933-6. [PubMed: 1848033].

3. Hallahan DE, Vogelzang NJ, Borow KM, Bostwick DG, Simon MA. Cardiac metastases from soft-tissue sarcomas. J Clin Oncol. 1986;4(11):16629. [PubMed: 3772419].

4. Khoo V, Ngan S, Guiney M, Lim-Joon D. Acute vascular embolus resulting from metastatic endocardial involvement with synovial sarcoma: report of a case and review of the literature. Australas Radiol. 1997;41(1):49-52. [PubMed: 9125069].
5. Frazier AA, Franks TJ, Pugatch RD, Galvin JR. From the archives of the AFIP: Pleuropulmonary synovial sarcoma. Radiographics. 2006;26(3):923-40. doi: 10.1148/rg.263055211. [PubMed:16702463].

6. Etienne-Mastroianni B, Falchero L, Chalabreysse L, Loire R, Ranchere D, Souquet PJ, et al. Primary sarcomas of the lung: a clinicopathologic study of 12 cases. Lung Cancer. 2002;38(3):283-9. [PubMed: 12445750].

7. Zeren H, Moran CA, Suster S, Fishback NF, Koss MN. Primary pulmonary sarcomas with features of monophasic synovial sarcoma: a clinicopathological, immunohistochemical, and ultrastructural study of 25 cases. Hum Pathol. 1995;26(5):474-80. [PubMed: 7750931].

8. Miettinen M, Limon J, Niezabitowski A, Lasota J. Calretinin and other mesothelioma markers in synovial sarcoma: analysis of antigenic similarities and differences with malignant mesothelioma. Am JSurg Pathol. 2001;25(5):610-7. [PubMed: 11342772]. 